\title{
HALL CURRENT EFFECT ON RADIATING SPAN-WISE FLUCTUATING MHD CONVECTIVE FLOW THROUGH POROUS MEDIUM IN A VERTICAL POROUS CHANNEL
}

\author{
Krishan Dev Singh ${ }^{1 *}$ and Alphonsa Mathew ${ }^{2}$ \\ ${ }^{1}$ Wexlow, Lower Kaithu, Shimla 171003, India \\ E-mail: kdsinghshimla@gmail.com \\ ${ }^{2}$ Department of Mathematics, St. Thomas College, Thrissur, Kerala-680001, India \\ E-mail: alphonsastc@gmail.com
}

(Received September 30, 2014)

\begin{abstract}
An analysis of an unsteady MHD convective flow of an electrically conducting viscous incompressible fluid through porous medium filled in a vertical porous channel is carried out. The two porous plates are subjected to a constant injection and suction velocity as shown in Fig. 1a, b. The temperature of the plate at $y^{*}=+\frac{d}{2}$ is assumed to be varying in space and time as $T^{*}\left(y^{*}, z^{*}, t^{*}\right)=T_{1}\left(y^{*}\right)+\left(T_{2}-\right.$ $\left.T_{1}\right) \cos \left(\frac{\pi z^{*}}{d}-\omega^{*} t^{*}\right)$. A magnetic field of uniform strength is applied perpendicular to the plates of the channel. The temperature difference between the plates is high enough to induce the heat due to radiation. It is also assumed that the conducting fluid is opticallythin gray gas, absorbing/ emitting radiation and non-scattering. The Hall current effects have also been taken into account. Exact solution of the partial differential equations governing the flow under the prescribed boundary conditions has been obtained for the velocity and the temperature fields. The primary and secondary velocities, temperature and the skin-friction and Nusselt number for the rate of heat transfer in terms of their amplitudes and phase angles have been shown graphically to observe the effects of suction parameter $\lambda$, Grashof number Gr, Hartmann number M, Hall parameter $H$, the permeability of the porous medium K, Prandtl number Pr, radiation parameter N, pressure gradient $\mathrm{A}$ and the frequency of oscillation $\omega$. The final results are then discussed in detail in the last section of the paper with the help of figures.
\end{abstract}

Keywords: Hall current, injection/suction, convective flow, magnetohydromagnetic (MHD), spanwise fluctuating, porous medium, radiation.

\section{INTRODUCTION}

The study of MHD free convection flow for electrically conducting fluid has attracted the attention of many scholars in view of its applications in many engineering problems such as meteorology, solar physics, motion of earth's core and chemical engineering etc. At the same time, these days the convective flows through porous medium is also receiving considerable attention due to its application in geophysical and astrophysical importance. The subject of geophysical dynamics nowadays has become an important branch of fluid dynamics due to the increasing interest to study environment. In astrophysics it is applied to study the stellar and solar structure, inter planetary and inter stellar matter, solar storms and 
flares etc. In engineering it finds its application in MHD generators, ion propulsion, MHD bearings, MHD pumps, MHD boundary layer control of re-entryof space vehicles etc. Literature related to hydromagnetic channel flows is reported by several scholars viz. CRAmmer and PAi [8], Ferraro and Plumpton [9], SherclifF [22] on account of their varied importance. MHD channel or duct flows are important from its practical point of view. ChANG and Lundgren [6] studied a hydromagnetic flow in a duct. YeN and CHANG [29] analyzed the effect of wall electrical conductance on the magnetohydrodynamic Couette flow. ATTIA [4] discussed unsteady MHD Couette flow of a viscoelastic fluid with heat transfer. AтTIA and Котв [5] investigated MHD flow between two parallel porous plates with heat transfer. HASSANIEN and MANSOUR [12] studied an unsteady magnetic flow through a porous medium between two infinite parallel plates.

In certain engineering devises the fluid such as gas ionized due to high temperature becomes electrical conductor. The ionized gas or plasma can be made to interact with the magnetic field. This interaction alters the heat transfer and friction characteristic. Nowadays many scholars have shown interest in studying the effect of magnetic field on the flow when the fluid is not only an electrical conductor but is capable of emitting and absorbing thermal radiation. AlAGOA et al. [2] have studied magnetohydrodynamic optically-transparent freeconvection flow with radiative heat transfer in porous media and time-dependent suction using an asymptotic approximation, showing that thermal radiation exerts a significant effect on the flow dynamics. MEBINE [15] investigated the radiation effects on MHD Couette flow with heat transfer between two parallel plates. GUPTA and GUPTA [10] investigated the effects of radiation on the free and forced convection of an electrically conducting fluid flowing inside an open ended vertical channel. HAKIEM [11] studied an oscillatory MHD flow on free convection radiation through a porous medium with constant suction. MAKINDE and MHONE [14] studied combined effects of transverse magnetic field and thermal radiation on MHD oscillatory flow in a channel filled with porous medium. SANYAL and ADHIKARI [20] analyzed heat radiation effect on MHD vertical channel. RAPTIS et al. [18] investigated Hydromagnetic free convection flow through a porous medium between two parallel plates. An exact solution of an unsteady periodic Poiseuille flow transpiration cooling and thermal radiation is obtained by SINGH [26]. MEBINE and GuMUS [16] investigated the steady MHD thermally radiating and reacting thermosolutal viscous flow through a channel with porous medium. SINGH and GARG [23] studied radiative heat transfer in MHD oscillatory flow through porous medium bounded by two vertical porous plates. VISKANTA [28] investigated the effects of transverse magnetic field on heat transfer to an electrically conducting and thermal radiating fluid flowing in a parallel channel. SINGH [25] analysed MHD mixed convection visco-elastic slip flow through a porous medium in a vertical porous channel with thermal radiation.

When the strength of the magnetic field is strong, one cannot neglect the effects of Hall current. The radiative convective flow of an electrically conducting fluid in the presence of a magnetic field is encountered in geophysical and cosmical fluid dynamics. It is also important in the solar physics involved in the sunspot development. In an ionized gas where the density is low and/or the magnetic field is very strong, the conductivity normal to the magnetic field is reduced due to the free spiraling of electrons and ions about the magnetic lines of force before suffering collisions and a current is induced in a direction normal to both the electric and the magnetic fields. This current, well known in the literature, is called the Hall currents. Due to Hall currents the electrical conductivity of the fluid becomes anisotropic 
and this causes the secondary flow. Hall effect is important when the Hall parameter, which is the ratio between the electron- cyclotron frequency and the electron-atom-collision frequency, is high. This happens when the magnetic field is high or when the collision frequency is low. Hall currents are of great importance in many astrophysical problems such as Hall accelerator and flight MHD as well as flows of plasma in a MHD power generator. REDDY et al. [19] presented Hall effects on MHD Couette flow through a porous straight channel. ATTIA [3] studied Hall current effects on velocity and temperature fields of an unsteady Hartmann flow. AHMED and ZUECO [1] obtained an exact solution to the problem of heat and mass transfer in a rotating vertical porous channel with Hall current. SINGH and KUMAR [27] analysed combined effects of Hall current and rotation on free convection MHD flow in a porous channel. The unsteady hydromagnetic free convection flow with Hall current and mass transfer along an accelerated porous plate with time dependent temperature and concentration has been studied by SATTAR and HossAin [21]. KINYANJUI et al.[13] have presented the magnetohydrodynamic free convection heat and mass transfer of a heat generating fluid past an impulsively started infinitely long vertical porous plate with Hall current and radiation absorption. SINGH et al. [24] have studied heat and mass transfer in an unsteady MHD free convective flow through a porous medium bounded by vertical porous channel in the presence of heat radiation and Hall current.

The aim of the present work is to analyse the MHD convective flow of viscous, incompressible and electrically conducting fluid through a porous medium filled in a vertical porous channel. The porous plates of the channel are subjected to constant injection and suction so that the fluid injected through one porous plate is sucked through the other with same velocity. A magnetic field of uniform strength is applied transverse to the channel plates. The temperature of one of the plates varies both in space and time. The Hall current effects have also been taken into account. A closed form solution of the partial differential equations governing the flow under the prescribed boundary conditions has been obtained for the velocity and the temperature fields. The final results are then discussed in the last section of the paper with the help of figures in detail.

\section{MATHEMATICAL FORMULATION OF THE PROBLEM}

Consider an unsteady flow of a viscous, incompressible and electrically conducting fluid through a highly porous medium in a vertical channel. The insulated plates of the channel are at distance ' $\mathrm{d}$ ' apart. The $\mathrm{X}^{*}$ - axis is oriented vertically upwards along the centreline of the channel. The $\mathrm{Y}^{*}$-axis taken perpendicular to the planes of the plates and a strong transverse magnetic field of uniform strength $\vec{B}=\left(0, B_{0}, 0\right)$ is applied along this axis. The porous walls of the vertical channel are lying in the $y^{*}= \pm \frac{d}{2}$ planes and the fluid is injected through the left porous plate with constant velocity (V) and simultaneously sucked through the other plate with the same velocity (V). As shown in Figure 1b, the spanwisecosinusoidal temperature of the plate at $y^{*}=+\frac{d}{2}$ varying both in space and time is assumed to be of the form

$$
T^{*}=T_{1}+\left(T_{2}-T_{1}\right) \cos \left(\frac{\pi z^{*}}{d}-\omega^{*} t^{*}\right) .
$$

Since the plates of the channel are of infinite extent, all the physical quantities except the pressure are independent of $\mathrm{x}^{*}$. The equation of Continuity $\nabla \cdot \vec{V}=0$ for the constant injection/suction at the plates of the channel integrates tov $v^{*}=V$, where $\vec{V}=\left(u^{*}, v^{*}, w^{*}\right)$ 
represents the velocity components in the directions $\left(\mathrm{X}^{*}, \mathrm{Y}^{*}, \mathrm{Z}^{*}\right)$ respectively. The physical configuration of the problem is shown in Figure $1 \mathrm{a} \& 1 \mathrm{~b}$

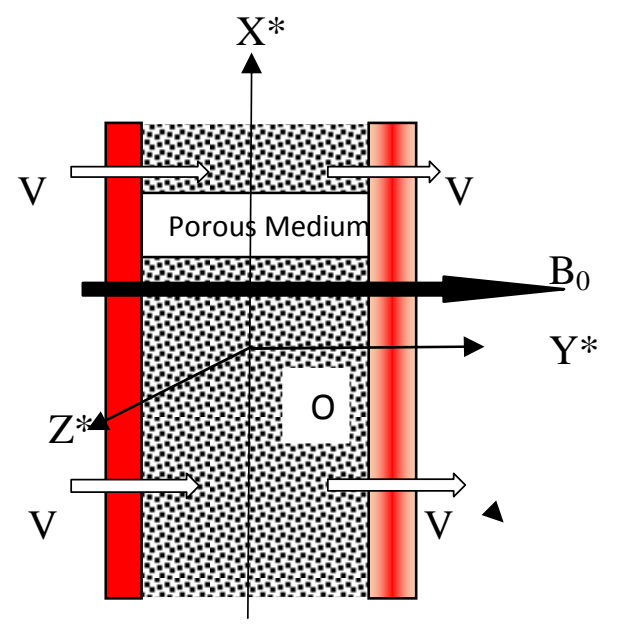

Fig. 1a. Hot vertical channel.

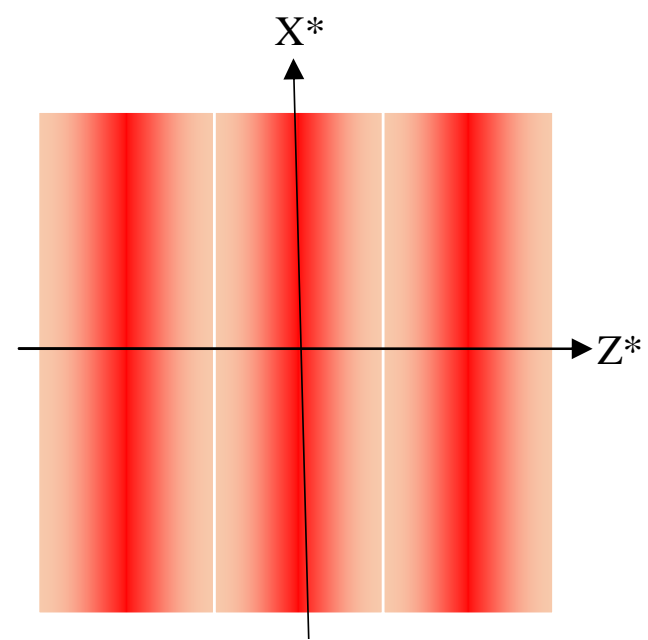

Fig. 1b. Spanwise cosinusoidal plate temperature.

The solenoidal relation for the magnetic field $\nabla \cdot \vec{B}=0$, gives $B_{y}^{*}=B_{0}$ (constant) where $\vec{B}=\left(B_{x}^{*}, B_{y}^{*}, B_{z}^{*}\right)$. Similarly if $\vec{J}=\left(J_{x}^{*}, J_{y}^{*}, J_{z}^{*}\right)$ are the components of electric current density $\vec{J}$ then the equation of conservation of electric charge gives, $J_{y}^{*}=$ constant. This constant is zero i.e. $J_{y}^{*}=0$ for the electrically non-conducting plates. Under the usual assumptions that the electron pressure (for a weakly ionized gas), the thermoelectric pressure, ion slip and the external electric field arising due to polarization of charges are negligible. Taking Hall current into account the generalized Ohm's law (COWLING [7]) is of the form

$\vec{J}+\frac{\omega_{e} \tau_{e}}{B_{0}}(\vec{\jmath} \times \vec{B})=\sigma\left(\vec{E}+\mu_{e} \vec{V} \times \vec{B}\right)$.

where $\vec{V}$ is the velocity vector, $\vec{B}$ is the magnetic field, $\vec{j}$ is the current density, $\vec{E}$ is the electric field, $\sigma$ is the electrical conductivity, $\mu_{e}$ is the magnetic permeability, $\omega_{e}$ is the cyclotron frequency, and $\tau_{e}$ is the electron collision time.

Under the usual assumptions that the electron pressure (for a weakly ionized gas), the thermoelectric pressure, ion slip and the external electric field arising due to polarization of charges are negligible. It is assumed that no applied and polarized voltage exists. This corresponds to the case where no energy being added or extracted from the fluid by electrical means (MEYER [17]) i.e. electric field $\vec{E}=0$. Therefore, equation (2) takes the form:

$$
\vec{J}+\frac{\omega_{e} \tau_{e}}{B_{0}}(\vec{J} \times \vec{B})=\sigma(\vec{V} \times \vec{B})
$$

Taking into account $J_{y}^{*}=0$, equation (3) can be written in component form as

$$
j_{x}^{*}-\omega_{e} \tau_{e} j_{z}^{*}=-\sigma B_{0} w^{*} \quad j_{z}^{*}+\omega_{e} \tau_{e} j_{x}^{*}=\sigma B_{0} u^{*},
$$

Solving equations (4) for $\mathrm{j}_{\mathrm{x}}^{*}$ and $\mathrm{j}_{\mathrm{z}}^{*}$, we get

$j_{x}^{*}=\frac{\sigma B_{0}}{\left(1+H^{2}\right)}\left(H u^{*}-w^{*}\right) \quad \operatorname{and} j_{z}^{*}=\frac{\sigma B_{0}}{\left(1+H^{2}\right)}\left(u^{*}+H w^{*}\right)$, 
where $\mathrm{H}=\omega_{\mathrm{e}} \tau_{\mathrm{e}}$ is the Hall parameter.

Thus, within the frame work of these assumptions and taking into account the usual Boussinsq's approximation the magnetohydrodynamic (MHD) forced and free convection flow through porous medium in the vertical porous channel in the presence of Hall current and thermal radiation is governed by the following momentum and energy differential equations:

$$
\begin{aligned}
& \frac{\partial u^{*}}{\partial t^{*}}+V \frac{\partial u^{*}}{\partial y^{*}}=-\frac{1}{\rho} \frac{\partial p^{*}}{\partial x^{*}}+\vartheta\left(\frac{\partial^{2} u^{*}}{\partial y^{* 2}}+\frac{\partial^{2} u^{*}}{\partial z^{* 2}}\right)-\frac{\sigma B_{0}^{2}\left(u^{*}+H w^{*}\right)}{\rho\left(1+H^{2}\right)}-\frac{\vartheta}{K^{*}} u^{*}+g \beta\left(T^{*}-T_{1}\right), \\
& 0=-\frac{1}{\rho} \frac{\partial p^{*}}{\partial y^{*}}, \\
& \frac{\partial w^{*}}{\partial t^{*}}+V \frac{\partial w^{*}}{\partial y^{*}}=-\frac{1}{\rho} \frac{\partial p^{*}}{\partial z^{*}}+\vartheta\left(\frac{\partial^{2} w^{*}}{\partial y^{* 2}}+\frac{\partial^{2} w^{*}}{\partial z^{* 2}}\right)-\frac{\sigma B_{0}^{2}\left(w^{*}-H u^{*}\right)}{\rho\left(1+H^{2}\right)}-\frac{\vartheta}{K^{*}} w^{*} \\
& \rho c_{p}\left(\frac{\partial T^{*}}{\partial t^{*}}+V \frac{\partial T^{*}}{\partial y^{*}}\right)=k\left(\frac{\partial^{2} T^{*}}{\partial y^{* 2}}+\frac{\partial^{2} T^{*}}{\partial z^{* 2}}\right)-\frac{\partial q^{*}}{\partial y^{*}},
\end{aligned}
$$

where $\vartheta$ is the kinematic viscosity. The last term in equation (9) stands for heat due to radiation and is given by

$$
\frac{\partial q^{*}}{\partial y^{*}}=4 a^{*} \sigma^{*}\left(T^{* 4}-T_{1}^{4}\right)
$$

for the case of an optically thin gray gas. Here $a^{*}$ is the mean absorption coefficient and $\sigma^{*}$ is Stefan- Boltzmann constant. We assume that the temperature differences within the flow are sufficiently small such that $\mathrm{T}^{* 4}$ may be expressed as a linear function of the temperature. This is accomplished by expanding $\mathrm{T}^{* 4}$ in a Taylor series about $\mathrm{T}_{1}$ and neglecting higher order terms, thus

$$
T^{* 4} \cong 4 T_{1}^{* 3} T^{*}-3 T_{1}^{4}
$$

Substituting (11) into (10) and simplifying, we obtain

$$
\frac{\partial q^{*}}{\partial y^{*}}=16 a^{*} \sigma^{*} T_{1}^{3}\left(T^{*}-T_{1}\right)
$$

The substitution of equation (12) into the energy equation (9) for the heat due to radiation, we get

$$
\rho c_{p}\left(\frac{\partial T^{*}}{\partial t^{*}}+V \frac{\partial T^{*}}{\partial y^{*}}\right)=k\left(\frac{\partial^{2} T^{*}}{\partial y^{* 2}}+\frac{\partial^{2} T^{*}}{\partial z^{* 2}}\right)-16 a^{*} \sigma^{*} T_{1}^{3}\left(T^{*}-T_{1}\right) .
$$

Equation (7) shows the constancy of the hydrodynamic pressure along the axis perpendicular to the plates. The boundary conditions for the problem are

$$
\begin{aligned}
& y^{*}=-\frac{d}{2}: \quad u^{*}=w^{*}=0, \quad T^{*}=T_{1}, \\
& y^{*}=\frac{d}{2}: \quad u^{*}=w^{*}=0, \quad T^{*}=T_{1}+\left(T_{2}-T_{1}\right) \cos \left(\frac{\pi z^{*}}{d}-\omega^{*} t^{*}\right)
\end{aligned}
$$

Where $\omega^{*}$ is the frequency of oscillations.

Introducing the following non-dimensional quantities

$$
x, y, z=\frac{x^{*}, y^{*}, z^{*}}{d}, u, w=\frac{u^{*}, w^{*}}{V}, \theta=\frac{T^{*}-T_{1}}{T_{2}-T_{1}}, t=\frac{t^{*} V}{d}, \omega=\frac{\omega^{*} d}{V}, p=\frac{p^{*}}{\rho V^{2}},
$$

into equations (6), (8) and (13), we get 


$$
\begin{aligned}
& \lambda\left(\frac{\partial u}{\partial t}+\frac{\partial u}{\partial y}\right)=-\lambda \frac{\partial p}{\partial x}+\left(\frac{\partial^{2} u}{\partial y^{2}}+\frac{\partial^{2} u}{\partial z^{2}}\right)-\frac{M^{2}(u+H w)}{\left(1+H^{2}\right)}-\frac{u}{K}+\operatorname{Gr} \theta, \\
& \lambda\left(\frac{\partial w}{\partial t}+\frac{\partial w}{\partial y}\right)=-\lambda \frac{\partial p}{\partial z}+\left(\frac{\partial^{2} w}{\partial y^{2}}+\frac{\partial^{2} w}{\partial z^{2}}\right)-\frac{M^{2}(w-H u)}{\left(1+H^{2}\right)}-\frac{w}{K}, \\
& \lambda \operatorname{Pr}\left(\frac{\partial \theta}{\partial t}+\frac{\partial \theta}{\partial y}\right)=\left(\frac{\partial^{2} \theta}{\partial y^{2}}+\frac{\partial^{2} \theta}{\partial z^{2}}\right)-N^{2} \theta,
\end{aligned}
$$

where ' $*$ ' represents the dimensional physical quantities,

$\lambda=\frac{V d}{\vartheta}$ is the injection/suction parameter,

$M=B_{0} d \sqrt{\frac{\sigma}{\mu}}$ is the Hartmann number,

$H=\omega_{e} \tau_{e}$ is the Hall parameter,

$K=\frac{K^{*}}{d^{2}}$ is the permeability of the porous medium,

$G r=\frac{g \beta d^{2}\left(T_{2}-T_{1}\right)}{\vartheta V}$ is the Grashof number,

$\operatorname{Pr}=\frac{\mu c_{p}}{k}$ is the Prandtl number,

$N=4 d \sqrt{\frac{a^{*} \sigma^{*} T_{1}^{3}}{k}}$ is the radiation parameter.

The boundary conditions in the dimensionless form become

$$
\begin{aligned}
& y=-\frac{1}{2}: u=w=0, \theta=0, \\
& y=\frac{1}{2}: u=w=0, \theta=\cos (\pi z-\omega t) .
\end{aligned}
$$

\section{SOLUTION OF THE PROBLEM}

In order to obtain the solution of this unsteady problem we combine equations (17) and (18) into a single equation by introducing a complex function of the form, $F=u+i w$, we get

$$
\lambda\left(\frac{\partial F}{\partial t}+\frac{\partial F}{\partial y}\right)=-\lambda\left(\frac{\partial}{\partial x}+i \frac{\partial}{\partial z}\right) p+\left(\frac{\partial^{2} F}{\partial y^{2}}+\frac{\partial^{2} F}{\partial z^{2}}\right)-\frac{M^{2}(1-\iota H)}{\left(1+H^{2}\right)} F-K^{-1} F+G r \theta .
$$

For convenience sake let us adopt complex variable notations for velocity, temperature and pressure. Thus, we write velocity, temperature and pressure as

$$
F(y, z, t)=F_{0}(y) e^{i(\pi z-\omega t)}, \theta(y, z, t)=\theta_{0}(y) e^{i(\pi z-\omega t)},-\left(\frac{\partial}{\partial x}+i \frac{\partial}{\partial z}\right) p=A e^{i(\pi z-\omega t)}
$$

where $\mathrm{A}$ is a constant. The boundary conditions in equations (20) and (21) can also be written in complex notations as

$$
\begin{aligned}
& y=-\frac{1}{2}: F=0, \theta=0, \\
& y=\frac{1}{2}: F=0, \theta=e^{i(\pi z-\omega t)} .
\end{aligned}
$$


Substituting expressions (23) into equations (22) and (19), we get

$$
\begin{aligned}
& \frac{d^{2} F_{0}}{d y^{2}}-\lambda \frac{d F_{0}}{d y}-\left\{\frac{M^{2}(1-\iota H)}{\left(1+H^{2}\right)}+\pi^{2}+K^{-1}-i \omega \lambda\right\} F_{0}=-\lambda A-G r \theta_{0}, \\
& \frac{d^{2} \theta_{0}}{d y^{2}}-\lambda \operatorname{Pr} \frac{d \theta_{0}}{d y}-\left(\pi^{2}+N^{2}-i \omega \lambda \operatorname{Pr}\right) \theta_{0}=0,
\end{aligned}
$$

with transformed boundary conditions

$$
\begin{aligned}
& y=-\frac{1}{2}: \quad F_{0}=0, \quad \theta_{0}=0, \\
& y=\frac{1}{2}: \quad F_{0}=0, \quad \theta_{0}=1 .
\end{aligned}
$$

The ordinary differential equations (26) and (27) are solved under boundary conditions (28), (29) and the solutions for the velocity and temperature fieldsare obtained, as

$$
\begin{aligned}
& F(y, z, t)=\left[\begin{array}{c}
\frac{G r}{2 \sinh \left(\frac{m-n}{2}\right)}\left[\begin{array}{c}
\left(\frac{e^{\frac{r-s}{2}}}{C_{1}}-\frac{e^{-\frac{r-s}{2}}}{C_{2}}\right)\left(e^{m y-\frac{n}{2}}-e^{n y-\frac{m}{2}}\right) \\
+\left(\frac{C_{1}-C_{2}}{C_{1} C_{2}}\right)\left(e^{m y+\frac{n}{2}}-e^{n y+\frac{m}{2}}\right) e^{-\frac{\lambda P r}{2}}
\end{array}\right\} \\
+\frac{2 \lambda A\left(e^{m y} \sinh \frac{n}{2}-e^{n y} \sinh \frac{m}{2}\right)}{\left\{\frac{M^{2}(1-\iota H)}{\left(1+H^{2}\right)}+\pi^{2}+K^{-1}-i \omega \lambda\right\}} \\
+\frac{\lambda A}{\left\{\frac{M^{2}(1-\iota H)}{\left(1+H^{2}\right)}+\pi^{2}+K^{-1}-i \omega \lambda\right\}}-\frac{G r}{2 \sinh \left(\frac{r-s}{2}\right)}\left(\frac{e^{r y-\frac{s}{2}}}{C_{1}}-\frac{e^{s y-\frac{r}{2}}}{C_{2}}\right)
\end{array}\right] e^{i(\pi z-\omega t),} \\
& (y, z, t)=\left(\frac{e^{r y-\frac{s}{2}}-e^{s y-\frac{r}{2}}}{2 \sinh \left(\frac{r-s}{2}\right)}\right) e^{i(\pi z-\omega t)} \text {, }
\end{aligned}
$$

where

$$
\begin{aligned}
& m=\frac{\lambda+\sqrt{\lambda^{2}+4\left\{\frac{M^{2}(1-\imath H)}{\left(1+H^{2}\right)}+\pi^{2}+K^{-1}-i \omega \lambda\right\}}}{2}, \quad n=\frac{\lambda-\sqrt{\lambda^{2}+4\left\{\frac{M^{2}(1-\iota H)}{\left(1+H^{2}\right)}+\pi^{2}+K^{-1}-i \omega \lambda\right\}}}{2}, \\
& r=\frac{\lambda P r+\sqrt{\lambda^{2} P r^{2}+4\left(\pi^{2}+N^{2}+i \omega \lambda P r\right)}}{2}, \quad s=\frac{\lambda P r-\sqrt{\lambda^{2} P r^{2}+4\left(\pi^{2}+N^{2}+i \omega \lambda P r\right)}}{2}, \\
& C_{1}=r^{2}-\lambda r-\left\{\frac{M^{2}(1-\iota H)}{\left(1+H^{2}\right)}+\pi^{2}+K^{-1}-i \omega \lambda\right\}, \\
& C_{2}=s^{2}-\lambda s-\left\{\frac{M^{2}(1-\iota H)}{\left(1+H^{2}\right)}+\pi^{2}+K^{-1}-i \omega \lambda\right\} .
\end{aligned}
$$

From the velocity field solution (30) we can obtain the skin-friction $\tau_{L}$ at the left wall in terms of its amplitude $|\mathrm{F}|$ and the phase angle as

$\tau_{L}=\left(\frac{\partial F}{\partial y}\right)_{y=-\frac{1}{2}}=|F| \cos (\pi z-\omega t+\varphi)$,

with 


$$
\begin{aligned}
F_{r}+i F_{i}=\frac{1}{2 \sinh \left(\frac{m-n}{2}\right)}\left[\begin{array}{c}
\frac{G r}{2 \sinh \left(\frac{r-s}{2}\right)}\left\{\begin{array}{c}
\left(\frac{e^{\frac{r-s}{2}}}{C_{1}}-\frac{e^{-\frac{r-s}{2}}}{C_{2}}\right)(m-n) e^{-\frac{\lambda}{2}}+ \\
\left(\frac{C_{1}-C_{2}}{C_{1} C_{2}}\right)\left(m e^{-\frac{m-n}{2}}-n e^{\frac{m-n}{2}}\right) e^{-\frac{\lambda P r}{2}}
\end{array}\right\} \\
+\frac{2 \lambda A\left(m e^{-\frac{m}{2}} \sinh \frac{n}{2}-n e^{-\frac{n}{2}} \sinh \frac{m}{2}\right)}{\left\{\frac{M^{2}(1-l H)}{\left(1+H^{2}\right)}+\pi^{2}+K^{-1}-i \omega \lambda\right\}}
\end{array}\right] . \\
-\frac{G r}{2 \sinh \left(\frac{r-s}{2}\right)}\left(\frac{r}{C_{1}}-\frac{s}{C_{2}}\right) e^{-\frac{\lambda P r}{2}} .
\end{aligned}
$$

The amplitude and the phase angle of the skin-friction are respectively given by

$$
|F|=\sqrt{F_{r}^{2}+F_{i}^{2}} \text {, and } \varphi=\tan ^{-1}\left(\frac{F_{i}}{F_{r}}\right) \text {. }
$$

Similarly, from the temperature field given in equation (31) the heat transfer coefficient $\mathrm{Nu}$ (Nusselt number) in terms of its amplitude $|H|$ and the phase angle $\psi$ can be obtained as

$N u=\left(\frac{\partial \theta}{\partial y}\right)_{y=-\frac{1}{2}}=\cos (\pi z-\omega t+\psi)$,

where $H_{r}+i H_{i}=\frac{(r-s) e^{-\frac{\lambda P r}{2}}}{2 \sinh \left(\frac{r-s}{2}\right)}$.

The amplitude $|H|$ and the phase angle $\psi$ of the heat transfer coefficient $\mathrm{Nu}$ (Nusselt number) are given by

$|H|=\sqrt{H r^{2}+H i^{2}}$ and $\psi=\tan ^{-1} \frac{H i}{H r}$ respectively.

\section{RESULTS AND DISCUSSION}

A closed form solution of the problem of magnetohydrodynamic convective flow in a vertical porous channel filled with porous medium is obtained. The two porous plates are subjected to constant injection and suction. The temperature of one of the channel plates varies span-wise cosinusoidally. It is also assumed that the electrically conducting fluid is optically-thin gray gas, absorbing/ emitting radiation and non-scattering. The analytical results obtained in the previous section are evaluated numerically for different sets of values of the parameters involved in the flow field. In order to have a better insight of the influence of parameters on the velocity and temperature fields these numerical values are then illustrated through figures. The influence of each of the parameters on the physical quantities like the velocity, the temperature, the amplitude and the phase of the skin-friction are depicted through these figures.

The variations of the primary and secondary velocity components against the variable $y$ with respect to each of the flow parameters are presented in Figs. 2 to 10. The primary velocity $\mathrm{u}(\mathrm{y}, \mathrm{z}, \mathrm{t})$ is shown by smooth curves while the secondary velocity $\mathrm{w}(\mathrm{y}, \mathrm{z}, \mathrm{t})$ is shown by dotted curves. The effects of the injection/suction $\lambda$ on the velocity are presented in Fig 2 . 


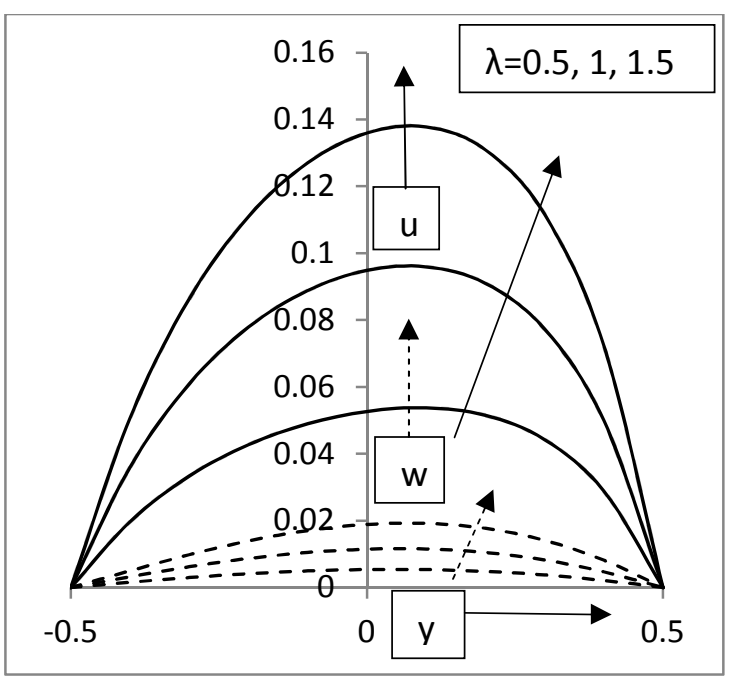

Fig. 2. Velocity profiles for $\mathrm{Gr}=1, \mathrm{M}=2, \mathrm{H}=1$, $\mathrm{K}=0.2, \operatorname{Pr}=0.7, \mathrm{~N}=1, \mathrm{~A}=2, \omega=1, \mathrm{z}=0.5$ and $\mathrm{t}=\pi / 2$.

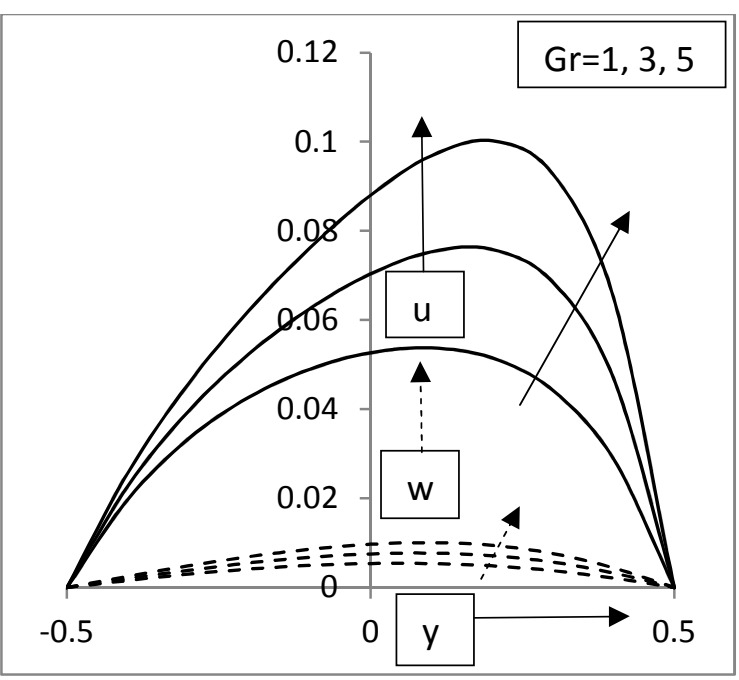

Fig. 3. Velocity profiles for $\lambda=0.5, \mathrm{M}=2, \mathrm{H}=1$, $\mathrm{K}=0.2, \operatorname{Pr}=0.7, \mathrm{~N}=1, \mathrm{~A}=2, \omega=1, \mathrm{z}=0.5$ and $\mathrm{t}=\pi / 2$.

It is quite obvious that with the increasing injection/suction both the primary and secondary velocities increase. Similarly the velocity behavior with the Grashof number is presented in Figure 3. It is also found from this figure that the primary velocity $\mathrm{u}(\mathrm{y}, \mathrm{z}, \mathrm{t})$ and the secondary velocity $w(y, z, t)$ increase with increasing Grashof number Gr. The maximum of the primary velocity profiles shifts toward right half of the channel due to the greater buoyancy force in this part of the channel because of the presence of hotter plate of the channel.

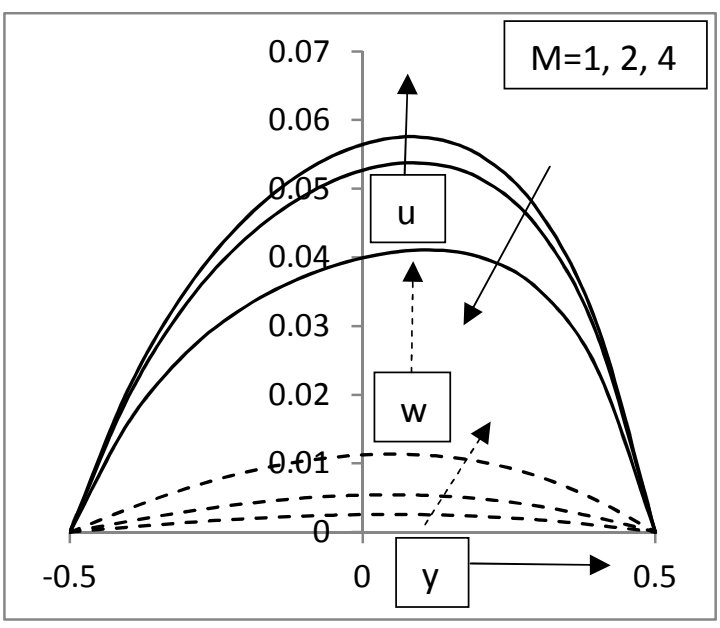

Fig. 4. Velocity profiles for $\lambda=0.5, \mathrm{Gr}=1, \mathrm{H}=1$, $\mathrm{K}=0.2, \operatorname{Pr}=0.7, \mathrm{~N}=1, \mathrm{~A}=2, \omega=1, \mathrm{z}=0.5$ and $\mathrm{t}=\pi / 2$.

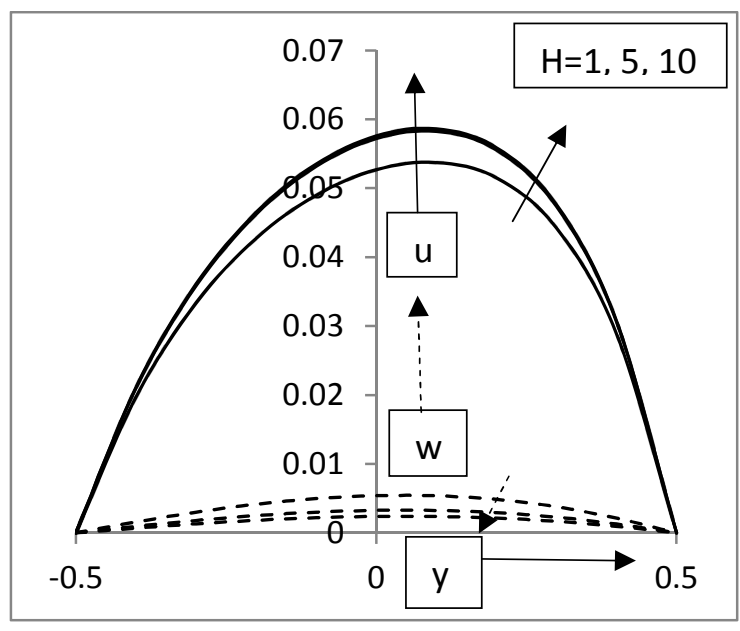

Fig. 5. Velocity profiles for $\lambda=0.5, \mathrm{Gr}=1, \mathrm{M}=2$, $\mathrm{K}=0.2, \operatorname{Pr}=0.7, \mathrm{~N}=1, \mathrm{~A}=2, \omega=1, \mathrm{z}=0.5$ and $\mathrm{t}=\pi / 2$.

The effect of the Hartmann number $M$ on the velocity field is exhibited in Fig. 4. The Hartmann number has opposing effects on the primary and secondary velocities. The primary velocity increases while the secondary velocity decreases with increasing Hartmann number M. This means that the Lorentz force due to the applied magnetic field has a dragging effect on $\mathrm{u}(\mathrm{y}, \mathrm{z}, \mathrm{t})$ while the secondary flow $\mathrm{w}(\mathrm{y}, \mathrm{z}, \mathrm{t})$ generated by the Hall current accelerates. Figure 5 shows that the increase of Hall parameter $\mathrm{H}$ increases the primary velocity but decreases the secondary velocity. The variations of the velocity profiles with the permeability of the porous medium $\mathrm{K}$ are presented in Fig. 6. It is noticed from this figure that the primary 
velocity and the secondary velocities increase with the increasing permeability of the porous medium K.

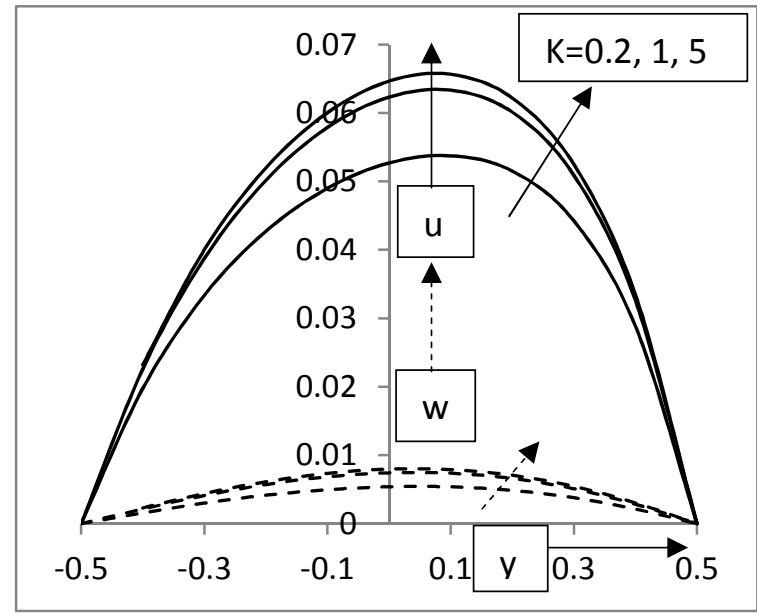

Fig. 6. Velocity profiles for $\lambda=0.5, \mathrm{Gr}=1, \mathrm{M}=2$, $\mathrm{H}=1, \operatorname{Pr}=0.7, \mathrm{~N}=1, \mathrm{~A}=2, \omega=1, \mathrm{z}=0.5$ and $\mathrm{t}=\pi / 2$.

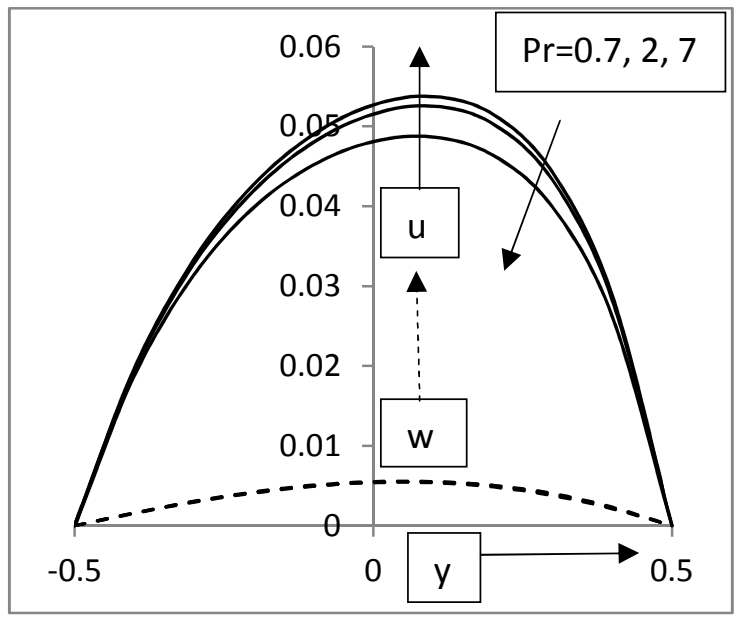

Fig. 7. Velocity profiles for $\lambda=0.5, \mathrm{Gr}=1, \mathrm{M}=2$, $\mathrm{H}=1, \mathrm{~K}=0.2, \mathrm{~N}=1, \mathrm{~A}=2, \omega=1, \mathrm{z}=0.5$ and $\mathrm{t}=\pi / 2$.

Physically it means that the resistance posed by the porous matrix reduces with increasing permeability which leads to the increase of $u(y, z, t)$ and $w(y, z, t)$. The effects of Prandtl number on the velocity are shown in Fig.7. The two values of $\operatorname{Pr}(=0.7$ and 7$)$ have been chosen to represent real fluids i.e. air and water respectively. It is inferred from this figure that with the increase of Prandtl number Pr the primary velocity decreases, however, there is no significant effect on the secondary velocity. The primary velocity is less in water $(\operatorname{Pr}=7)$ than in air $(\operatorname{Pr}=0.7)$. The variation of the velocity profiles with the radiation parameter $\mathrm{N}$ is presented in Fig.8. The primary velocity and the secondary velocity decrease with increasing radiation parameter N. From Fig.9 it is evident that the primary velocity $u(y, z, t)$ and the secondary velocity $\mathrm{w}(\mathrm{y}, \mathrm{z}, \mathrm{t})$ go on increasing with the increasing favourable pressure gradient A $(>0)$. It is also observed from Fig.10 that the primary velocity decreases with increasing frequency $\omega$ and secondary velocity first increase as the frequency of oscillations increase from 1 to 5 but then it reverses for $\omega=10$.

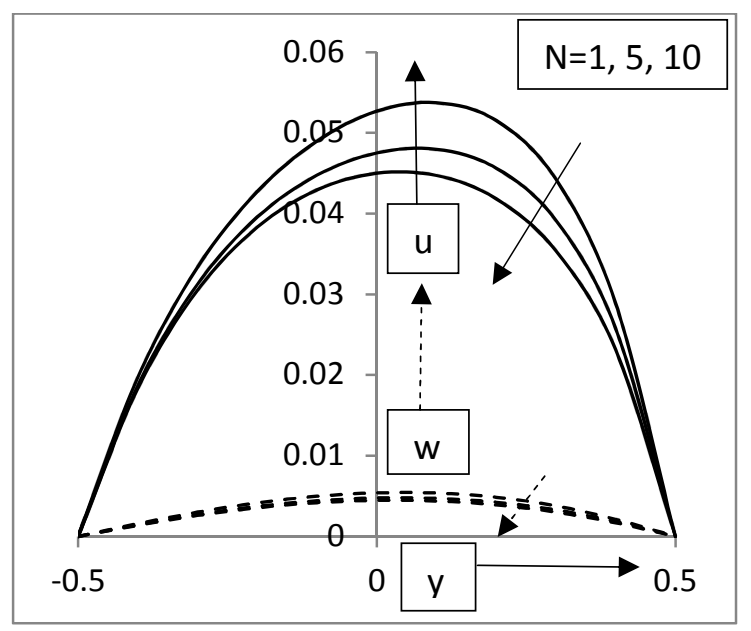

Fig. 8. Velocity profiles for $\lambda=0.5, \mathrm{Gr}=1, \mathrm{M}=2$, $\mathrm{H}=1, \mathrm{~K}=0.2, \operatorname{Pr}=0.7, \mathrm{~A}=2, \omega=1, \mathrm{z}=0.5$ and $\mathrm{t}=\pi / 2$.

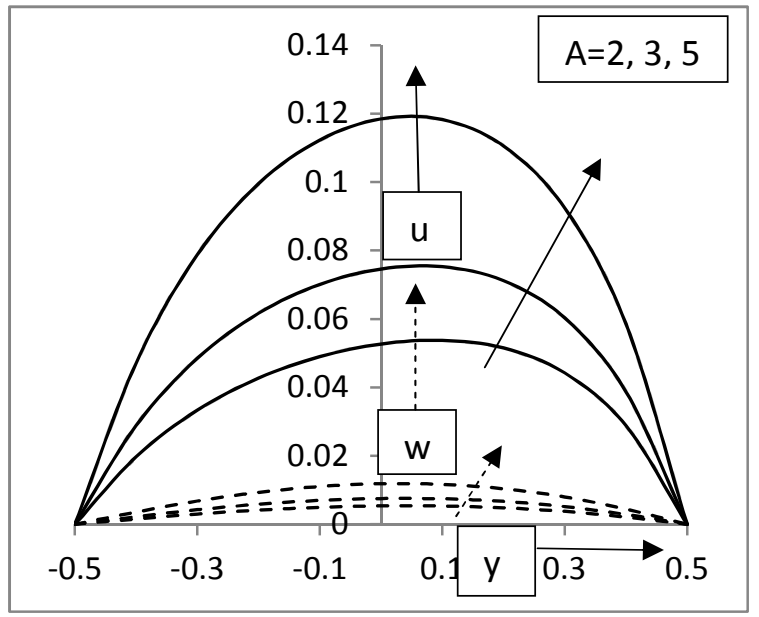

Fig. 9. Velocity profiles for $\lambda=0.5, \mathrm{Gr}=1, \mathrm{M}=2$, $\mathrm{H}=1, \mathrm{~K}=0.2, \mathrm{Pr}=0.7, \mathrm{~N}=1, \omega=1, \mathrm{z}=0.5$ and $\mathrm{t}=\pi / 2$. 


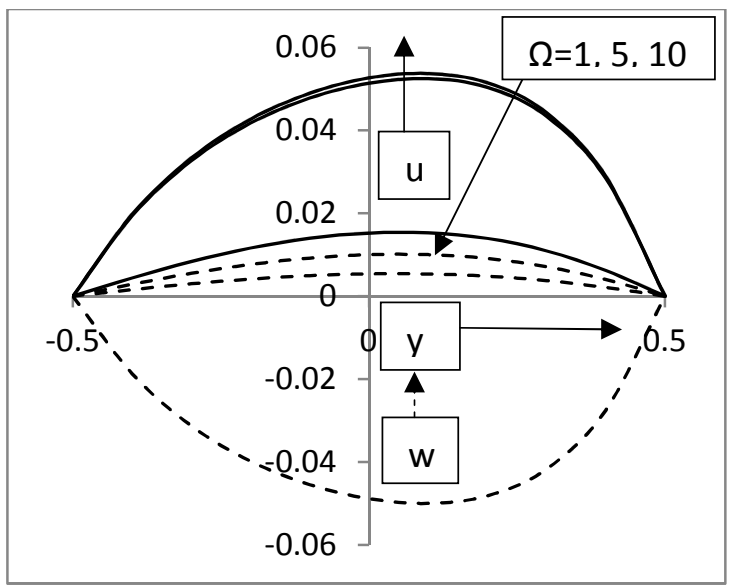

Fig. 10. Velocity profiles for $\lambda=0.5, \mathrm{Gr}=1, \mathrm{M}=2$, $\mathrm{H}=1, \mathrm{~K}=0.2, \operatorname{Pr}=0.7, \mathrm{~N}=1, \mathrm{~A}=2, \mathrm{z}=0.5$ and $\mathrm{t}=\pi / 2$.
Table 1. Sets of parameter values plotted in Figs. $11 \& 12$.

$\begin{array}{lllllllll}\lambda & \text { Gr M } & \mathbf{H} & \mathbf{K} & \text { Pr } & \text { N } & \text { A } & \text { Curves } \\ 0.5 & 1 & 2 & 1 & 0.2 & 0.7 & 1 & 2 & \text { I }(---) \\ 1.0 & 1 & 2 & 1 & 0.2 & 0.7 & 1 & 2 & \text { II } \\ 0.5 & 5 & 2 & 1 & 0.2 & 0.7 & 1 & 2 & \text { III } \\ 0.5 & 1 & 4 & 1 & 0.2 & 0.7 & 1 & 2 & \text { IV } \\ 0.5 & 1 & 2 & 5 & 0.2 & 0.7 & 1 & 2 & \text { V } \\ 0.5 & 1 & 2 & 1 & 1.0 & 0.7 & 1 & 2 & \text { VI } \\ 0.5 & 1 & 2 & 1 & 0.2 & 7.0 & 1 & 2 & \text { VII } \\ 0.5 & 1 & 2 & 1 & 0.2 & 0.7 & 5 & 2 & \text { VIII } \\ 0.5 & 1 & 2 & 1 & 0.2 & 0.7 & 1 & 3 & \text { IX }\end{array}$

The skin-friction in terms of its amplitude $|\mathrm{F}|$ and phase angle has been shown in Figs. 11 and 12 respectively. The sets of values of parameters listed in Table 1 are presented graphically in these figures. The effect of each of the parameter on $|\mathrm{F}|$ and is assessed by comparing each curve with dotted curves in these figures. In Fig.11 the comparison of curves II, III, V, VI and IX with dotted curve I (---) indicates that the amplitude increases with the increase of injection/suction parameter $\lambda$, Grashof number Gr, Hall parameter $H$, the permeability of the porous medium $\mathrm{K}$ and the pressure gradient parameter $\mathrm{A}$. It is expected physically also because due to the increase of these parameters the primary velocity increases and consequently the faster flows give rise to more skin-friction. Similarly, the comparison of other curves IV, VII and VIII with the dotted curve I (---) depicts that the skin-friction amplitude decreases with the increase of Hartmann number M, Prandtl number Pr and the radiation parameter $\mathrm{N}$ because the primary velocity due to these parameters decreases and for slow flows skin-friction is less. It is obvious that amplitude $|\mathrm{F}|$ remains almost constant with increasing frequency of oscillations.

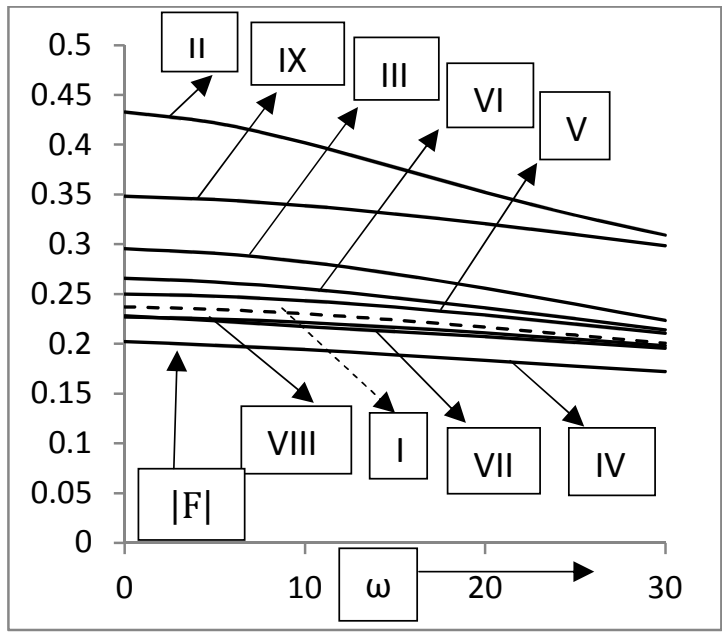

Fig. 11. Amplitude of skin-friction.

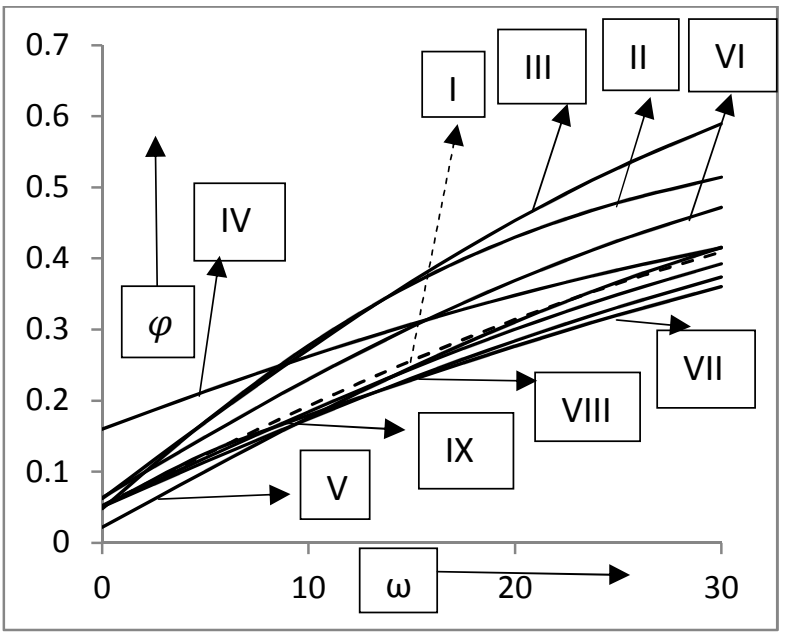

Fig. 12. Phase angle of skin-friction.

It is clear from Figure 12 showing the variations of the phase angle $\varphi$ of the skinfriction that there is always a phase lead because the values of $\varphi$ remain positive throughout. Here again the comparison of curves II, III, IV and VI with the dotted curve I (---) indicates that the phase increases with the increase of injection/suction parameter $\lambda$ Grashof number Gr, 
Hartmann number $M$ and the permeability of the porous medium $\mathrm{K}$ respectively. The comparison of the rest of the curves namely V, VII, VIII and IX with dotted curve I reveals that the phase angle decreases with the increase of Hall parameter $\mathrm{H}$, Prandtl number Pr, radiation parameter $\mathrm{N}$ and the pressure gradient $\mathrm{A}$. It is also noticed from this figure that the phase lead goes on increasing with increasing frequency of oscillations $\omega$.

Figure 13 illustrates the variation of the temperature field with the variation of different flow parameters. The comparison of different curves with the dotted curve reveals that there is a decrease in temperature with increasing parameters like the injection/suction $\lambda$ or the Prandtl number Pr or the radiation parameter $\mathrm{N}$ or frequency of oscillations $\omega$.

The amplitude $|H|$ and the phase angle $\psi$ of the rate of heat transfer against the frequency of oscillations $\omega$ are illustrated in Figures 14 and 15 respectively. It is evident from Fig. 15 that the amplitude $|H|$ decreases with the increase of injection/suction parameter $\lambda$, Prandtl number Pr and the radiation parameter N. The amplitude in the case of water $(\operatorname{Pr}=7)$ becomes negligible for larger values of oscillations $\omega$. The increase of radiation parameter stabilizes the amplitude with increasing frequency of oscillations $\omega$. It is noticed from Fig.15 that the phase lead with increasing radiation remains linear with the increasing frequency of oscillations $\omega$. A decrease in phase angle $\psi$ is also observed with the increase of radiation parameter $\mathrm{N}$. The phase angle of the Nusselt number starts oscillating between the phase lag and the phase lead as the injection/suction parameter or the Prandtl number are increased. For the values of injection/suction parameter the amplitude remains linear initially for smaller oscillations but oscillates thereafter for larger $\omega$.

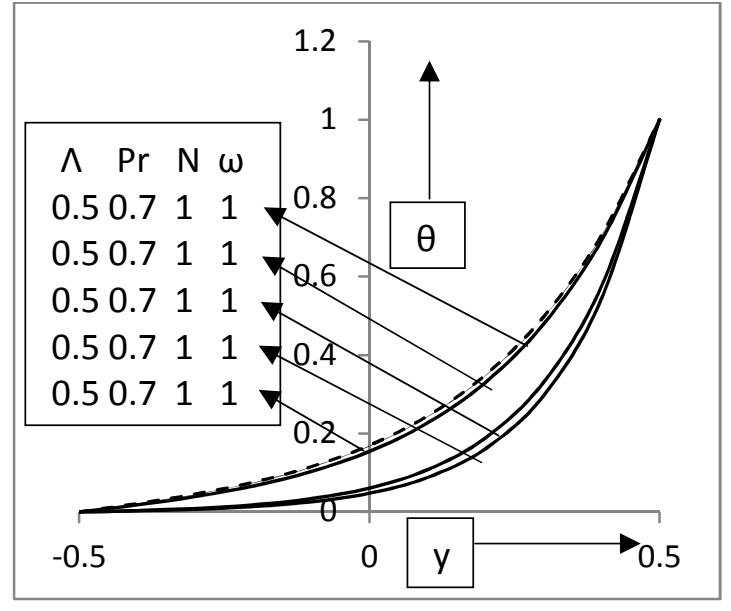

Fig. 13. Temperature profiles for $\mathrm{z}=0.5, \mathrm{t}=\pi / 2$.

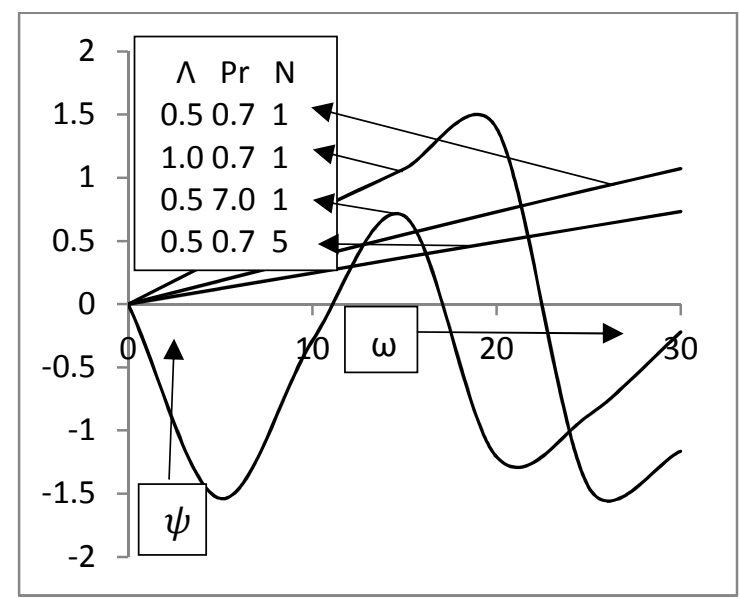

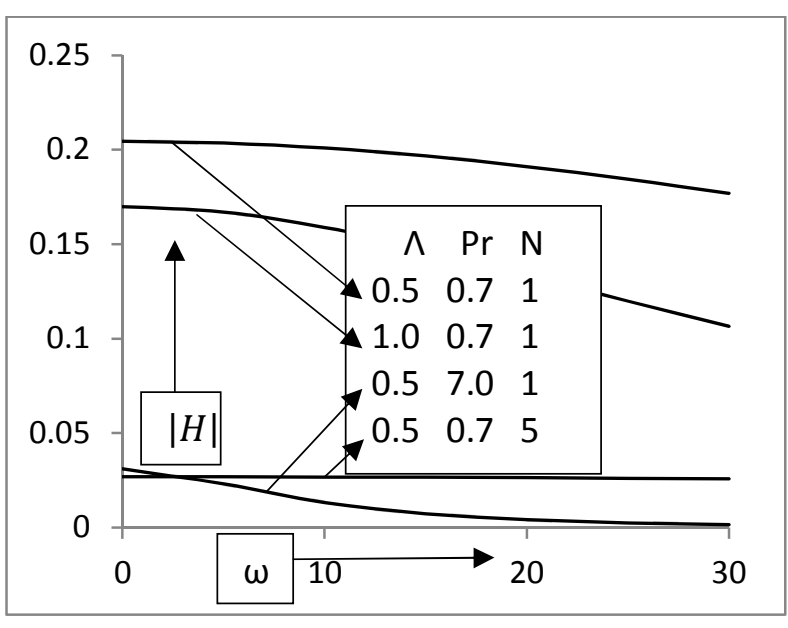

Fig. 14. Amplitude of Nusselt number.

Fig. 15. Phase angle of Nusselt number. 


\section{CONCLUSIONS}

An unsteady hydromagnetic convective flow of viscous incompressible and electrically conducting fluid in a vertical porous channel is investigated when the temperature of one of the plates varies spanwise cosinusoidally. The entire system consisting of channel plates and the fluid rotates about an axis perpendicular to the plates. A closed form solution of the problem is obtained. Following features are concluded from the mathematical analysis:

(i) The primary velocity $u(y, z, t)$ and the secondary $w(y, z, t)$ both increase with the increase of the injection/suction parameter $\lambda$, Grashof number Gr, permeability of the porous medium $\mathrm{K}$ and the favorable pressure gradient $\mathrm{A}$.

(ii) There are opposing effects on primary and secondary velocities due to the increasing Hartmann number $M$, Hall parameter that is, $u(y, z, t)$ and $w(y, z, t)$ decrease and increase respectably.

(iii) Due to increasing radiation primary and secondary velocities decrease.

(iv) The flow in the channel reverses for large frequency of oscillations $\omega$.

(v) The amplitude $|\mathrm{F}|$ of the skin-friction increases due to the increase of those parameters because of which the primary and secondary velocities increase.

(vi) Similarly, the amplitude $|\mathrm{F}|$ of the skin-friction decreases due to the increase of those parameters because of which the primary velocity decreases.

(vii) In other words faster flows lead to more skin-friction and for slow flows skin-friction is less.

(viii) The temperature and the amplitude of the rate of heat transfer decrease with the increase of any of the parameters that appear.

(ix) The phase of the rate of heat transfer oscillates between phase lag and lead for increasing injection/suction parameter and the Prandtl number.

\section{References:}

[1] Ahmed, S. and Zueco, JoAquin, Modelling of heat and mass transfer in a rotating vertical porous channel, Chem. Eng. Comm. 198 (2011) 1294-1308.

[2] Alagon, K.D. TAy, G. and AbBEy, T.M., Radiative and free convective effects of a MHD flow through a porous medium between infinite parallel plates with timedependent suction, Astrophysics and Space Science 260 (1999) 455-468.

[3] AtTIA, H.A., Hall current effects on velocity and temperature fields of an unsteady Hartmann flow, Can. J. Phys. 76 (1998) 739.

[4] AtTiA, H.A., Unsteady MHD Couette flow of a viscoelastic fluid with heat transfer, Kragujevac J. Sci. 32 (2010) 5-15.

[5] AттіA, H.A. and Котв, N.A., MHD flow between two parallel plates with heat transfer, ActaMechanica 117 (1996) 215-220.

[6] Chang, C.C. and Lundgren, T.S., Duct flow in magnetohydrodynamics, ZAMP 12 (1961) 100-114.

[7] Cowling, T.G., Magnetohydrodynamics, Interscience Publications Inc., New York, USA (1957).

[8] CRAmmer, K.R. and PAI, S.I., Magneto fluid dynamics for engineers and applied physicists, McGraw-Hill Book Co. New York (1973). 
[9] Ferraro, V.C.A. and Plumpton, C., An Introduction to Magneto Fluid Mechanics. Clarandon Press, Oxford (1966).

[10] GuPTA, P.S. and GUPTA, A.S., Radiation effect on hydrodynamic convection in a vertical channel, Int. J. Heat and Mass Transfer 17 (1974) 1437-1442.

[11] HAKIEM, E.I.M.A., MHD oscillatory flow on free convection radiation through porous medium with constant suction velocity, J. Magnetism and Magnetic Materials 220 (2000) 271-276.

[12] Hassanien, I.A. and Mansour, M.A., Unsteady magnetic flow through a porous medium between two infinite parallel plates, Astrophysics and Space science $\mathbf{1 6 3}$ (1990) 241- 246.

[13] Kinyanjui, M., Chartuverdi, N. and UpPal, S.M., Magnetohydrodynamic free convection heat and mass transfer of a heat generating fluid past an impulsively started infinite vertical porous plate with Hall current and radiation absorption, Energy Convers. Manage. 42 (8) (2001) 917-931.

[14] Makinde, O.D. and Mhone, P.Y., Heat transfer to MHD oscillatory flow in a channel filled with porous medium, Rom. Journ. Phys. 50 (2005) 931-938.

[15] Mebine, P., Radiation effects on MHD Couette flow with heat transfer between two parallel plates, Global J. Pure Appl. Math. 3 (2007) 191-202.

[16] Mebine, P. and Gumus, R.H., On steady MHD thermally radiating and reacting thermosolutal viscous flow through a channel with porous medium. Hindawi Publishing Corporation, Int. J. of Mathematics and Mathematical Sciences (2010) doi: $10.1155 / 2010 / 287435$.

[17] MEYER, R.C., On reducing aerodynamic heat transfer rates by MHD techniques, $J$. Aerospace Sci. 25 (1958) 561-563.

[18] Raptis, A. Massalas, C. and Tizvanidis, G., Hydromagnetic free convection flow through a porous medium between two parallel plates, Physics Letters A 90 (1982) 288289.

[19] Reddy, N. Bhaskara and BATHAiah, D., Hall effects on MHD Couette flow through a porous straight channel, Def. Sci. J. 32 (1982) 313-326.

[20] SANYAL, D.C. and ADHIKARI, A., Effects of radiation on MHD vertical channel flow, Bull. Cal. Math. Soc. 98 (2006) 487-497.

[21] SatTAR, M.A. and Hossain, M.M., Unsteady hydromagnetic free convection flow with Hall current and mass transfer along an accelerated porous plate with time dependent temperature and concentration, Can. J. Phys. 70 (1992) 369-375.

[22] SherClifF, A.J., A text Book of Magnetohydrodynamics, Pergamon Press Ltd. New York (1965).

[23] Singh, K.D. and GARG, B.P., Radiative heat transfer in MHD oscillatory flow through porous medium bounded by two vertical porous plates, Bull. Cal. Math. Soc. 102 (2010) 129-138.

[24] Singh, K.D., Khem Chand and Sharma, Shavnam, Heat and Mass transfer in an unsteady MHD free convective flow through a porous medium bounded by vertical porous channel in the presence of radiative heat and Hall current, Int. J. Math. Sci. \& Engng. Appl. 6 (2012) 317-336.

[25] SingH, K.D., MHD mixed convection visco-elastic slip flow through a porous medium in a vertical porous channel with thermal radiation. Kragujevac J. Sci. 35 (2013) 27-40.

[26] SingH, K.D., Rotating oscillatory MHD Poiseuille flow: An exact solution, Kragujevac J. Sci. 35 (2013) 15-25.

[27] Singh, K.D. and Kumar, RAKesh, Combined effects of Hall current and rotation on free convection MHD flow in a porous channel, Indian J. Pure \& Appl. Phys. 47 (2009) 617-623. 
[28] VisKanTA, R., Effects of transverse magnetic field on heat transfer to an electrically conducting and thermal radiating fluid flowing in a parallel channel, ZAMP 14 (1963) 353-361.

[29] Yen, J.T. and Chang, C.C., Magnetohydrodynamic Couette flow as affected by wall electrical conductances, ZAMP 15 (1964) 400. 